\title{
NON-LINEAR VIBRATIONS OF THREE-LAYER BEAMS WITH VISCOELASTIC CORES, II : EXPERIMENT
}

\author{
M. W. HYER \\ Department of Engineering Science ana echanics, \\ Virginia Polytechnic Institute and State University, \\ Blacksburg, Virginia 24061 U.S.A.
}

W. J. ANDERSON

Department of Aerospace Engineering, University of Michigan

AND

R. A. ScotT

Department of Applied Mechanics and Engineering Science, University of Michigan, Ann Arbor, Michigan 48109, U.S.A.

(Received 8 February 1978, and in revised form 20 May 1978)

\begin{abstract}
Experimental results are presented for large amplitude, forced motion of damped, three-layer beams. The beams are constructed with a viscoelastic material constrained between stiff, elastic, outer layers. The sandwich beam is axially restrained; therefore large amplitude displacements cause non-linear response. When the beam is forced at one-half of the lateral vibration resonant frequency, superharmonic response occurs. The experiment is briefly described and frequency response characteristics, spatial shapes and a measure of superharmonic response are presented. The results are compared with predictions from a previously developed theory.
\end{abstract}

\section{INTRODUCTION}

In previous work Kovac, Anderson and Scott [1] gave theoretical and experimental results on frequency response and mode shapes for the large amplitude, lateral motion of three-layer sandwich beams. The beams had viscoelastic cores, identical thin metallic face sheets and clamped ends which were restrained from moving toward each other. Due to the axial restraint, the large amplitude motion induced non-linear response. Experimentally, the forcing mechanism was a magnet, positioned to one side of the beam, with a sinusoidal current in the coils. The response of the beam was measured with a non-contacting inductive probe which was positioned on the opposite side of the beam. As was done by Bennett and Eisley [2] in their work on homogeneous beams, the theoretical force was taken to be a pure sinusoid with a frequency twice that of the coil current. In this procedure the constant force on the beam, an effect felt to be small, was ignored.

On the whole, reasonable agreement between theory and experiment was reported in reference [1]. An exception to this agreement occurred, however, when the beam was forced at a frequency in the vicinity of one-half the linear natural frequency. Near this frequency discrepancies arose between theory and experiment in both frequency response and mode shape.

The differences seemed to be connected with the appearance of a strong superharmonic component in the response. Since the problem was non-linear, the resulting theoretical investigations by those authors were based on an approximate method in which a single 
spatial mode shape and a time response which was harmonic with the same period as the forcing frequency were used. The response of the beam was predicted by evaluating the complex moduli of the viscoelastic core at the forcing frequency. Unfortunately, the observed behavior near one-half the linear natural frequency could not be predicted with this assumed spatial and temporal dependence. The main problem with the theory was its inability to handle viscoelasticity when more than one frequency was present.

In reference [3], which is a companion paper to this one, the current authors presented a theory in which the difficulty with the multiple frequency response was overcome through use of the heredity integral and its relation to complex moduli. Other noteworthy features of the extended theory are its ability to handle unsymmetric beams and thick face sheets. As effected by using Galerkin's technique and the method of harmonic balance, and with allowance for response at the forcing frequency as well as at twice the forcing frequency, the numerical solution of fourteen non-linear, simultaneous, algebraic equations gave theoretical frequency response information which was compared with the experimental results of Kovac, Anderson and Scott. Even though the constant force in the input was not neglected in this new theory, considerable deviation between theory and experiment still existed, as is shown in Figure 4 of reference [3].

At this juncture, a review of the earlier experimental set-up, particularly regarding the electromagnetic forcing, was in order. Because electromagnetic forcing involves no mechanical contact, it has been widely used. However, there are pitfalls associated with electromagnetic forcing. Basically, in a magnetic material, the non-linear relation between the magnetic intensity $B$ and the magnetic induction $H$ (which is linearly proportional to the coil current) can cause harmonics of the current frequency to appear in the force produced. In addition, the force produced by a magnet varies inversely with distance from the end of the magnet. Thus, as the beam vibrates, it moves through a spatially non-uniform force field. These two effects can combine to produce a force on the beam which is substantially different from that of a pure sinusoid. This distortion in force undoubtedly will be reflected in the response of the beam. If the system being forced is linear and if the distortion is known, the effects of the distortion can be accounted for in the response. However, if the system being forced is non-linear, it is not an easy matter to account for the effects of the distortion in the response. In fact, the effects may obscure any non-linear response or be misinterpreted as a non-linear phenomenon.

An additional potential problem involved the inductive probe. Non-contacting inductive probes, which work only for electrically conducting materials, operate by creating high frequency eddy currents near the surface of the object whose displacement is being measured. These eddy loops create magnetic fields which interact with the original field in the probe and, since this interaction is a function of distance between the object and the tip of the probe, an unknown distance can be determined by prior calibration. The calibration of the probe depends on the conductivity of the object being measured and if the conductivity changes, for example, due to temperature change, the calibration is changed. In addition to the temperature effect, the present authors found that if a ferromagnetic material is subject to an extraneous magnetic field, the calibration of the transducer for that material depends on the strength of the magnetic field. Therefore, since the experiment of Kovac, Anderson and Scott was done with beams having identical ferromagnetic face sheets, there is a strong possibility that when the inductive probe was used to measure mode shape along the length of the beam, the magnetic field produced by the forcing device influenced the probe response.

With the possibility of unwanted harmonics in the force time history and the chance that the probe was effected by the magnetic forcing device, there was room for further experimental investigation. Considerable effort was made to eliminate, or at least minimize, the unwanted harmonics in the force by using feedback to control the harmonic content of the 
force on the beam. This effort is discussed in reference [4]. To eliminate the interaction of the magnetic beam material with the inductive probe, unsymmetric beams were fabricated. The unsymmetric beams were made with one face-layer of ferromagnetic material, for forcing purposes, and the other face-layer of metallic, but not magnetic, material for the purpose of measuring beam response with the inductive probe. The amplitude of the beam displacement was measured as a function of forcing frequency and the spatial shape was measured at various frequencies. In addition, the harmonic content of the beam response was found. The remainder of this paper describes the experimental set-up, presents the experimental results and compares the results with predictions from the extended theory.

\section{EXPERIMENT}

The beams tested were $330 \mathrm{~mm}$ long, $25.4 \mathrm{~mm}$ wide and of various thicknesses. The results reported here are for beams $2.08 \mathrm{~mm}$ thick. The face-sheet nearer the forcing device was made of $0.254 \mathrm{~mm}$ ferromagnetic shim stock and the other was made of $0.254 \mathrm{~mm}$ springhardness phosphor bronze. The layers were bonded together with a single stage cyanacrylic adhesive. To prevent crushing of the neoprene core when the beams were mounted in the fixture to simulate clamped end conditions, the core in the ends of the beam in contact with the support mechanism was replaced by brass fillers. The fillers on each end were $38 \mathrm{~mm}$ long and thus the actual length of the beam between clamped supports was $254 \mathrm{~mm}$. The beam support fixture, previously used in references [1] and [2], was a modified lathe bed. A schematic of the fixture is shown in Figure 1 while Figure 2 shows the actual set-up. Steel end pieces, made of $152 \mathrm{~mm} \times 114 \mathrm{~mm} \times 102 \mathrm{~mm}$ blocks, were bolted to the lathe bed to provide the end support. Within each end piece was a set of steel jaws for clamping. Since the beams were to be restrained from axial stretching, two $25.4 \mathrm{~mm} \times 76.2 \mathrm{~mm}$ steel bars were bolted between the tops of the end pieces to give the support fixture additional rigidity. Mounting the beam specimens in the support fixture involved aligning the beam in the clamping mechanism and tightening various bolts in the clamps and axial support bars. During this mounting procedure it was possible to induce extraneous initial axial loads or small initial curvature in the beams. To minimize these effects, back-to-back axial strain gauges were mounted on the beam to keep a check on these initial conditions. In general, it was possible to keep the initial strains and curvatures small. The lathe bed was chosen as a basis for experiments since the lathe tool mount provided a convenient mechanism for mounting the beam displacement measuring probe. With this set-up, the probe could accurately traverse the length of the beam for monitoring mode shapes or it could remain stationary at any particular point along the beam to measure displacement time histories.

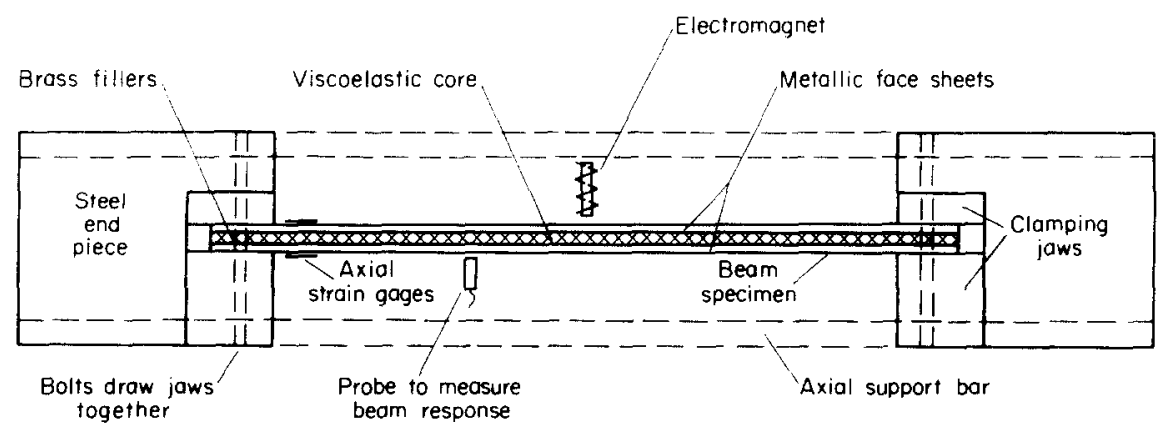

Figure 1. Schematic of beam support fixture. 


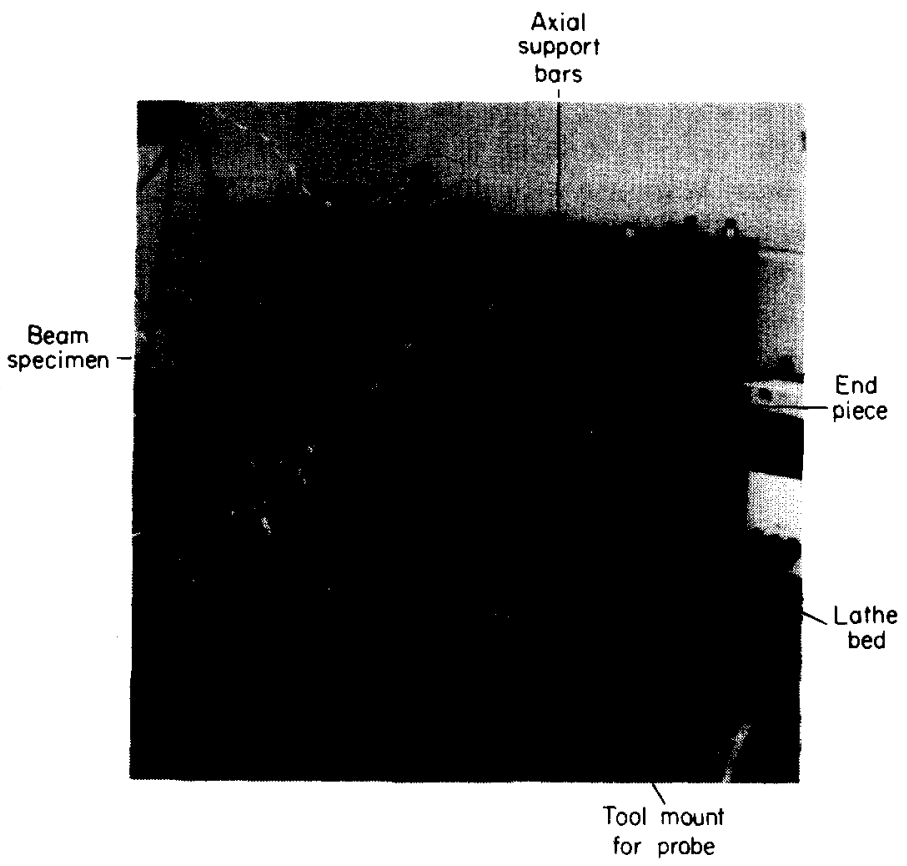

Figure 2. View of experimental set-up.

As explained in reference [4], the time history of force from an electromagnet with sinusoidal current in the coils is actually a constant force, $F_{0}$, plus an oscillatory component with amplitude $F_{1}$. With feedback incorporated in the electromagnetic forcing system to minimize the effects of unwanted harmonics, the constant force could be controlled independently of the oscillatory component. Thus the tests were conducted with a certain level of constant force on which was superimposed a certain level of oscillatory force.

\section{EXPERIMENTAL RESULTS}

Experimental and theoretical response curves for two different levels of forcing are shown in Figures 3 and 4 . The rms displacement at midspan is plotted as a function of $\Omega / \bar{\omega}_{R}$, where

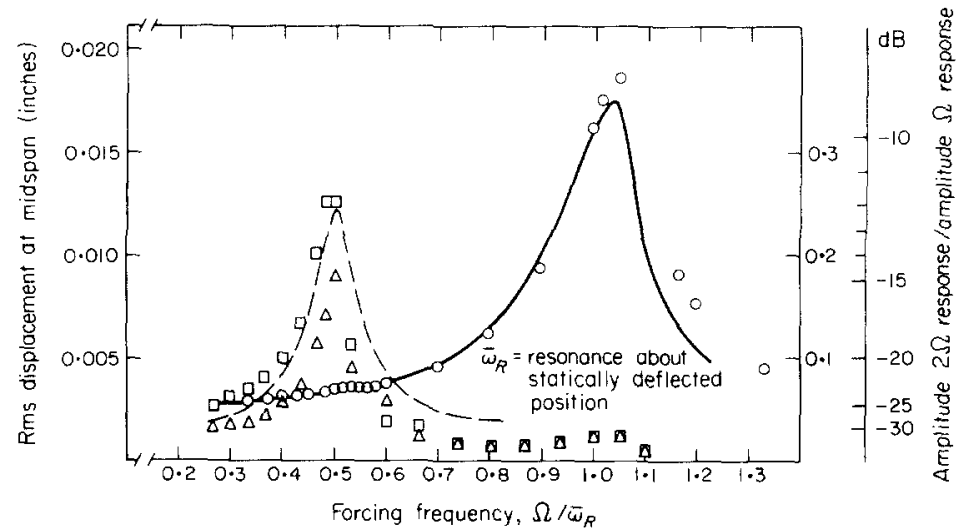

Figure 3. Frequency response of beam, $F_{0}=0.67 \mathrm{~N}, F_{1}=0.22 \mathrm{~N} . \square$, Ratio of amplitudes, experimental, without feedback; $\triangle$, ratio of amplitudes, experimental, with feedback; 0 , rms displacement, experimental; - - , ratio of amplitudes, theory; - - , rms displacement, theory. (Face-sheet thickness $=1 \mathrm{in}=2.54 \mathrm{~cm}$.) 


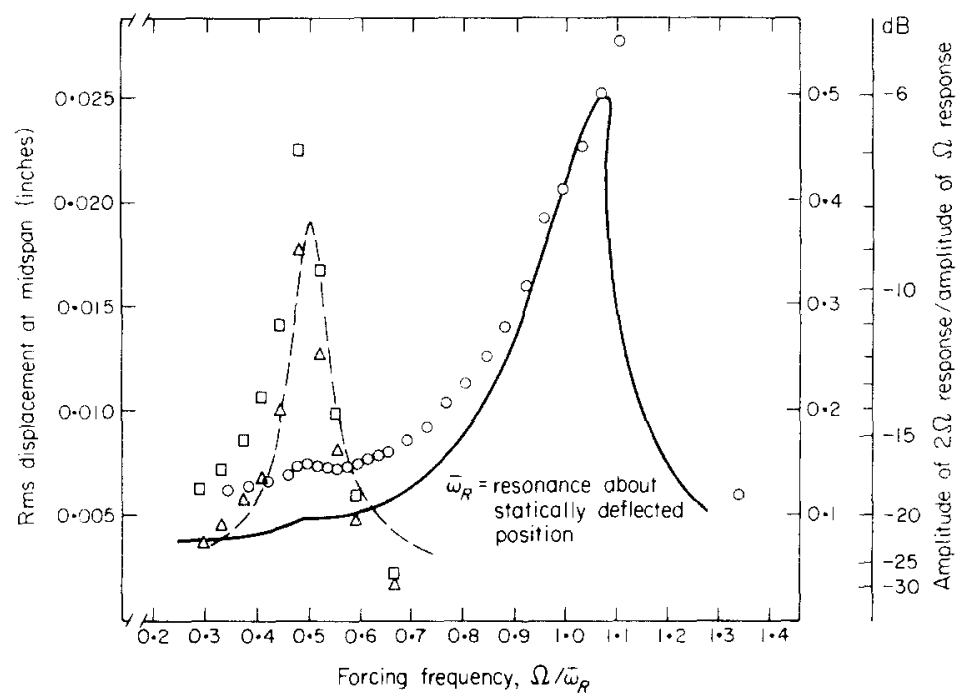

Figure 4. Frequency response of beam, $F_{0}=0 \cdot 80 \mathrm{~N}, F_{1}=0.32 \mathrm{~N}$. Key as Figure 3.

$\bar{\omega}_{R}$ is the natural frequency of small vibration about the statically deflected position of the beam, due to the constant component of the force, $F_{0}$, and $\Omega$ is the forcing frequency. In both cases the static force was chosen larger in magnitude than the oscillatory force, $F_{1}$, so as to have a more pronounced superharmonic response. Also shown on each figure is the ratio of the $2 \Omega$ superharmonic component of response to the $\Omega$ component of response. This ratio was determined by using an on-line spectrum analyser. The $2 \Omega$ component is of considerable interest since it was the unexpected occurrence of this response in reference [1] that prompted the present investigation. By virtue of the design of the feedback forcing system, the corrective feedback to reduce unwanted harmonics in the force could temporarily be eliminated and the open loop distortions could be studied. Comparison of the feedback and no-feedback beam responses indicates to what degree the distortion in the force caused a $2 \Omega$ component of response in the beam. Without feedback, the non-linear $2 \Omega$ component of the beam response could erroneously be overestimated. The $2 \Omega$ component of response without corrective action in the forcing system is shown in the figures. As expected, the $2 \Omega$ component of response is larger without feedback. This is because without feedback, the $2 \Omega$ component of response is due to the actual non-linearity and the response due to the $2 \Omega$ component in the force. Comparison of the feedback and no-feedback cases is a measure of effectiveness of the control system on the force. Of course the $2 \Omega$ response with feedback is what should be compared with theory.

Figure 5 shows the assumed and experimental spatial shapes of the beam, normalized to the midspan deflection, as obtained while forcing the beam at the resonant frequency and

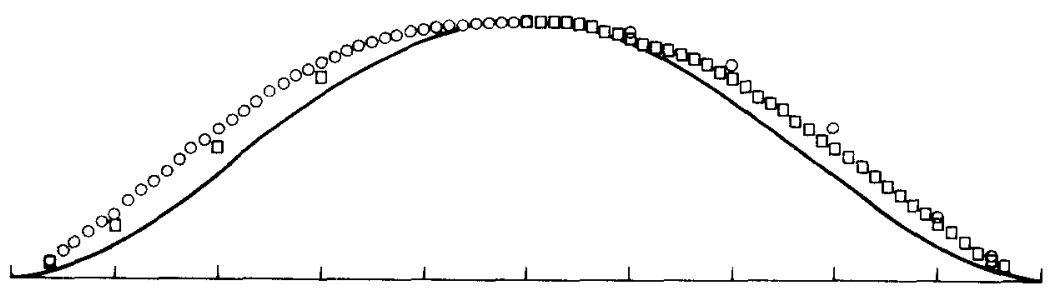

Figure 5. Spatial shape of beam, $F_{0}=0.80 \mathrm{~N}, F_{1}=0.32 \mathrm{~N}$, normalized at midspan. - - Assumed shape, $16\left(x^{2}-\frac{1}{4}\right)^{2},[3] ; 0$, forcing at resonant frequency, experimental; $\square$, forcing at one-half resonant frequency, experimental. 
at one-half the resonant frequency. The results shown in this figure are for the case of $F_{0}=0.8 \mathrm{~N}$ and $F_{1}=0.32 \mathrm{~N}$ but the shapes are similar at other forcing levels.

\section{DISCUSSION AND CONCLUSION}

The comparison between the theory of the companion paper [3] and this experiment is generally good, particularly at the lower forcing level. Theoretical predictions show superharmonic response could be expected when forcing the beam near one-half its linear natural frequency and experimental evidence confirms this. The spatial shape is independent of forcing frequency in this range and a single spatial mode representation is a good approximation. The magnitude of the superharmonic component is a function of forcing level, as evidenced by the noticeable increase in amplitude near one-half the natural frequency for the case with larger values of forcing.

For the large force level, the experimentally measured amplitudes of the beam displacement at midspan are greater than the theoretical predictions. In addition, the measured superharmonic response is somewhat less than predicted. Both of these effects are the result of unwanted support flexibility. The superharmonic response depends on the ability of the support mechanism to provide complete axial restraint of the beams. In the theory it was assumed that the support mechanism was perfectly rigid, an impossible ideal. Thus, with any axial flexibility, the non-linearity, and thus the superharmonic, would not be as pronounced. In addition, any flexibility of the support at the clamped ends, which allowed a small amount of rotation, would result in larger-than-predicted transverse displacements for any given forcing level. This effect would be more noticeable at the larger force levels. The lack of perfectly clamped end conditions also causes deviations between the experimentally measured and the assumed mode shapes. Both theoretical and experimental results have been normalized in Figure 5, but it is evident from the figure the experimental shapes indicate that deflections near the supports are larger than the predicted values. Part of the deviation is due to the shape used in the theory being an approximation to the actual shape. However, it is felt that a portion of the deviation is due to rotation of the end supports allowing larger deflections of the beam than a perfectly rigid support.

Despite these slight deviations, the comparison between theory and experiment was generally good. It can be concluded, for axially restrained beams such as these, that a measurable amount of superharmonic response is present, a single spatial mode shape is adequate over the frequency range tested and the response of the beam is predictable.

\section{REFERENCES}

1. E. J. Kovac, JR., W. J. Anderson and R. A. Scott 1971 Journal of Sound and Vibration 17, 25-39. Forced non-linear vibrations of a damped sandwich beam.

2. J. A. BeNNETt and J. G. EISLeY 1970 American Institute of Aeronautics and Astronautics Journal 8, 734-739. A multiple-degree-of-freedom approach to nonlinear beam vibrations.

3. M. W. Hyer, W. J. ANDerson and R. A. Scott 1976 Journal of Sound and Vibration 46, 121-136. Non-linear vibrations of three-layer beams with viscoelastic cores, I. Theory.

4. M. W. Hyer, W. J. ANderson and R. A. Scott 1978 Experimental Mechanics 18, 161-166. An electromagnetic forcing device. 